\title{
EDITORIAL
}

\section{The promise of cell-based therapy}

f recent media reports are to be believed, cell-based therapy could successfully treat Alport syndrome, offering hope to patients with this disease. Close examination of the data on which these reports are based, however, shows that the implications for human disease are in no way immediate. In their study, LeBleu and colleagues found that infusion of bone-marrow-derived cells into an experimental mouse model of Alport syndrome improved renal histology and survival (LeBleu, V. et al. J. Am. Soc. Nephrol. 20, 2112-2117; 2009). Although the findings of LeBleu et al. are intriguing and valuable in that they contribute to current understanding of the regenerative potential of the kidney, further investigation is certainly warranted before this technique makes the transition to clinical care.

The regenerative properties of stem cells have stimulated enormous interest in the potential of these cells to treat seemingly incurable conditions. The therapeutic potential of stem cells has already been realized for specific indications - for example, hematopoietic stem cell transplantation is a standard treatment for leukemia. However, the use of stem cell therapy for most indications is still largely experimental. The hype surrounding the promise of stem cells has nonetheless led to the emergence of stem cell clinics that offer unproven stem cell 'therapies' for a multitude of conditions including spinal cord injury and traumatic brain injury-conditions for which very little evidence of the safety and efficacy of stem cell therapy are available. Patients who access this treatment are not only paying for a treatment that may not work, but are also at risk of adverse effects such as tumor formation.

The use of such stem cell therapies also undermines the ethical and scientifically legitimate progress of stem cell research. The International Society for Stem Cell Research (ISSCR) condemns the unproven use of stem cells or their derivatives in series of patients outside of clinical trials. Their 2008 guidelines provide a framework for the responsible development of clinically useful stem cell therapies (http://www.isscr.org/clinical_trans/ pdfs/ISSCRGLClinicalTrans.pdf), and underscore the importance of independent ethical and scientific review throughout the translational research process. Of interest, in August 2009, the ISSCR's president, Irving Weissman, proposed that unproven stem cell therapies should be identified and named if the providers of such 'therapies' cannot provide the ISSCR with evidence of rigorous and independent scientific and ethical review (Weismann, I. Cell Stem Cell 5, 151-153; 2009).
Despite their conclusion that cell-based therapy is unequivocally a viable option for the treatment of Alport syndrome, LeBleu and colleagues also acknowledge that further understanding of the therapeutic potential of cell therapy is critical for testing in the clinic. For a start, better understanding of the mechanisms by which the implanted cells exert their effects is required. The researchers suggest that transdifferentiation or cell fusion may have a role; however, according to Weismann, no credible demonstration of stem cell transdifferentiation exists. Several experimental studies have demonstrated the ability of bone-marrow-derived cells to exert beneficial, mostly anti-inflammatory effects in different tissues, including the kidney. These effects are probably mediated by indirect mechanisms, such as, for example, the release of growth factors that elicit repair. Although evidence does suggest that the kidney has a greater capacity for repair than initially thought, the renal stem cell field currently lags behind that of other biological systems. Studies in experimental systems that demonstrate potential avenues for treatment in kidney disease should be applauded; however, it is of paramount importance that the realistic implications of these experiments are not exaggerated because of the risk that high expectations might not be fulfilled. And, as has been observed in other areas of stem cell biology, stem cell clinics will not wait for the efficacy and safety of treatments to be validated in clinical trials if public demand for these treatments exists.

Cell-based therapies could quite possibly revolutionize the treatment of genetic and functional kidney disorders; however, further basic research is required to improve our understanding of cell replacement in the adult kidney and how it changes in response to damage. Translational cell therapy studies need to be reviewed by independent, expert committees and the findings from such studies should be disseminated responsibly, without hype. Patients also need improved access to information about the realistic risks and benefits of such approaches to enable balanced and informed decisions to be made about any renal stem cell 'therapy' that is offered. The paper by LeBleu et al. is important because it contributes to our understanding of stem cell biology in the kidney. The questions left unanswered, however, accentuate the fact that we are not yet at a stage where these cells can be administered to patients with the certainty that they will be safe and beneficial.

doi:10.1038/nrneph.2009.209

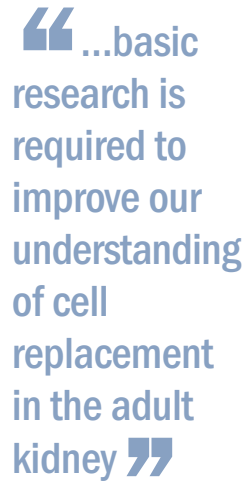

Susan J. Allison is the Editor of Nature Reviews Nephrology.

Competing interests The author declares no competing interests. 